\title{
Estudio de estrellas cefeidas en cúmulos galácticos
}

María de Jesús Quiróz*

\section{RESUMEN}

Se pretende elaborar una imagen tridimensional de la Nube Menor de Magallanes mediante la elaboración de una base de datos, datos obtenidos del sitio: http://www.astrouw.edu.pl/ ftp/ogle/. Este sitio tiene información de estrellas Cefeidas de la Nube Menor de Magallanes, datos que son organizados en una base de datos y que permiten el cálculo de la distancia a cada una de las estrellas que forman parte de la Nube Menor de Magallanes, para luego realizar la imagen tridimensional del grupo de estrellas seleccionado. Como resultado se obtiene una base de datos de estrellas cefeidas y su imagen tridimensional. El cálculo de las distancias se ha hecho utilizando el filtro visible, pero igual pueden utilizarse otras bandas del espectro electromagnético, de manera que puede ampliarse este resultado.

\section{Palabras clave: Nube Menor de Magallanes. Estrellas Cefeidas. distancias}

\section{ABSTRACT}

Is intended to produce a three-dimensional image of stars of the minor Magellanic Cloud through the development of a database, data obtained from the site: http://www.astrouw.edu.pl/ ftp/ogle/. This site has information star Cepheid of the minor Magellanic Cloud, data which are organized in a database and allow the calculation of the distance to each of the stars forming part of the minor Magellanic Cloud to then perform the three-dimensional selected Star group image. As a result gets a star cefeidas database and its three-dimensional image calculation of distances has been using the visible filter, but as other bands of the electromagnetic spectrum can be used so that you can extend this result.

\section{Key Words: Minor Magellanic Cloud. Star Cepheid.}

\footnotetext{
* María de Jesús Quiroz, mariatesis1@yahoo.com

Facultad de Ciencias Espaciales, Observatorio Astronómico Centroamericano de Suyapa,

Universidad Nacional Autónoma de Honduras.
} 


\section{INTRODUCCIÓN}

La determinación de distancias es un problema fundamental en Astrofísica. Si queremos saber acerca de las propiedades físicas de un objeto astronómico, una de las primeras cosas que debemos saber es su distancia.

Realmente no existe una forma directa y certera de determinar las distancias a las galaxias que están más allá de la Vía Láctea; los astrónomos determinan primero la distancia hasta las estrellas cercanas en nuestra galaxia con tanta precisión como les sea posible, y luego utilizan una serie de otras técnicas que llegan progresivamente más lejos en el espacio, para determinar las distancias que nos separan de los sistemas más distantes. A menudo, a este proceso se lo denomina como "la escala cósmica de distancias".

A lo largo de los años, se han encontrado varios estimadores diferentes de distancias. Uno de estos es una clase particular de estrellas conocidas como Cefeidas variables. Son utilizadas como uno de los primeros "escalones" de la escala cósmica de distancias. Las Cefeidas son estrellas muy luminosas y escasas, cuyo brillo varía en una forma muy regular. Se las denomina así por la estrella Delta Cephei en la constelación de Cefeo, que fue la primera estrella variable conocida de este tipo en particular, y que es lo suficientemente luminosa como para ser observada fácilmente a simple vista. Existe una relación, llamada ley períodoluminosidad, que vincula directamente la magnitud absoluta de una estrella cefeida, calculada en el máximo de su curva, con el valor de su período de pulsación.

El aumento de la luminosidad de las cefeidas en función del período, tomado de la relación período-luminosidad, es compatible con la teoría de la pulsación estelar según la cual la luminosidad depende del radio y, a su vez, este último es proporcional al período. La consecuencia más importante de la relación períodoluminosidad es que proporciona un método razonablemente seguro para evaluar la magnitud absoluta de una cefeida. Una vez conocida ésta, es posible conocer la distancia calculando la diferencia respecto a la magnitud aparente (módulo de distancia). Por este motivo, las cefeidas tienen también el importante papel de indicadores de distancia. Como tales, su importancia en astronomía para la medida de las distancias extragalácticas es enorme. Por ejemplo, identificar una cefeida en una galaxia distante y medir su período de pulsación permite conocer inmediatamente su distancia, y con ella, la de la galaxia misma.

Se plantea este proyecto con el objeto principal de obtener una imagen tridimensional de la Nube Menor de Magallanes y validar las estrellas cefeidas de 
esta galaxia como estándares de distancia, para lo cual se ha elaborado una base de datos conteniendo información de estrellas en cúmulos de esta galaxia; por ejemplo sus coordenadas ecuatoriales, su magnitud absoluta y aparente, el coeficiente de absorción y el período. Los datos han sido obtenidos del sitio: http://www.astrouw.edu.pl/ ftp/ogle/, se entra a Main OGLE results, y luego a Estrellas variables y se escoge Catalog of Cepheids from the SMC (2049 objects) de donde se obtiene el mapa interactivo para obtener los datos antes mencionados. Se ha obtenido la distancia a estas estrellas utilizando la relación PeríodoLuminosidad para eventualmente hacer un gráfico en tres dimensiones de la Nube Menor de Magallanes.

\section{METODOLOGÍA}

Fase 1: Búsqueda de bibliografía

Fase 2: Recolección y organización de datos

Fase 3: Elaboración de la base de datos

Fase 4: Cálculo de la distancia a las estrellas

Fase 5: Graficar en tres dimensiones las estrellas seleccionadas

\section{RESULTADOS}

1. Se obtuvo bibliografía principalmente The HST Key Project on the extragalactic distance scale XIV; un proyecto que utiliza los datos del Telescopio Espacial Hubble para calcular nuevas distancias a galaxias.

2. Se tomaron datos de más de 200 estrellas en cúmulos de la Nube Menor de Magallanes y se organizaron en una base de datos.

3. Una base de datos: Se ha elaborado una base de datos que contiene coordenadas ecuatoriales, período de pulsación, exceso de color, magnitud en el visible, magnitud aparente y la distancia a cada una de más de 200 estrellas.

4. La base de datos está hecha en Excell y contiene un programa que calcula distancia a las estrellas utilizando la relación Período-Luminosidad.

5. Un gráfico tridimensional de las estrellas seleccionadas; eje $x=$ ascensión recta, eje y= declinación; eje z= distancia.

\section{DISCUSIÓNYCONCLUSIONES}

Se ha elaborado una base de datos que contiene coordenadas ecuatoriales, período de pulsación, exceso de color, magnitud en el visible, magnitud aparente y la distancia a cada una de 194 estrellas. 
Para la elaboración de la base de datos, se utilizó un catálogo de Cefeidas con cerca de 34,000 BVI mediciones coleccionadas durante la fase OGLE-II en la modalidad de acceso interactivo. Se seleccionaron 194 objetos (estrellas Cefeidas clásicas) de los cuales se obtuvieron 6 parámetros: período, exceso de color, magnitud aparente, coeficiente de absorción, estos datos fueron necesarios para calcular la magnitud absoluta y eventualmente la distancia a la que se encuentran los objetos, también se tomaron los datos de Ascención Recta(AR) y declinación (Dec). para poder hacer el gráfico en tres dimensiones ( $x=A R, y=D e c, z=$ distancia .)

El gráfico de las estrellas de la Nube Mayor de Magallanes fue elaborado con el Software origin 8.

- Se prevé un pequeño error en el cálculo de las distancias, debido a la metalicidad de la estrellas cefeidas escogidas.

- El cálculo de las distancias se ha hecho utilizando el filtro visible, pero igual pueden utilizarse otras bandas del espectro electromagnético.

- Puede utilizarse la misma metodología para trabajar con estrellas RRlyra que también se consideran candelas estándar y confirmar así la validez de las estrellas Cefeidas como patrones estándar de distancia.

\section{BIBLIOGRAFÍA}

Silbermann et al. 1998. ApJ in press: astro-ph/9806017: The HST Key Project on the extragalactic distance scale XIV. The Cepheids in NGC 1365.

Hodge, Paul. 1988, Astronomical Society of the Pacific: The age Distribution and History of Formation of Large Magellanic Cloud Clusters.

Mendoza, E. E. 1956. Las ley de extinción interestelar en las Pléyades. ApJ. 123;54. Madore and freedman. 1991. The cepheid distance scale. PASP. 103;933.

Feast and Walker. 1987. Cepheids as distance indicators. ARAA. 25; 345.

Turner. 1986. Galactic clusters with associated cepheid variables. NGC 6087 and S Normae. AJ 92;111.

Johnsonand Mitchell. 1958. The color-magnitud of the Pléyades cluster. ApJ. 128;31 Udalski etal.1999. ActaAstron. 49; 223.

\section{Enlaces}

- http://www.astrouw.edu.pl/ ftp/ogle/

- http://vizier.u-strasbg.fr

- http://astro.estec.esa.nl/SA-general/Projects/Hipparcos/

- http://www.ipac.caltech.edu/HOkp/ 


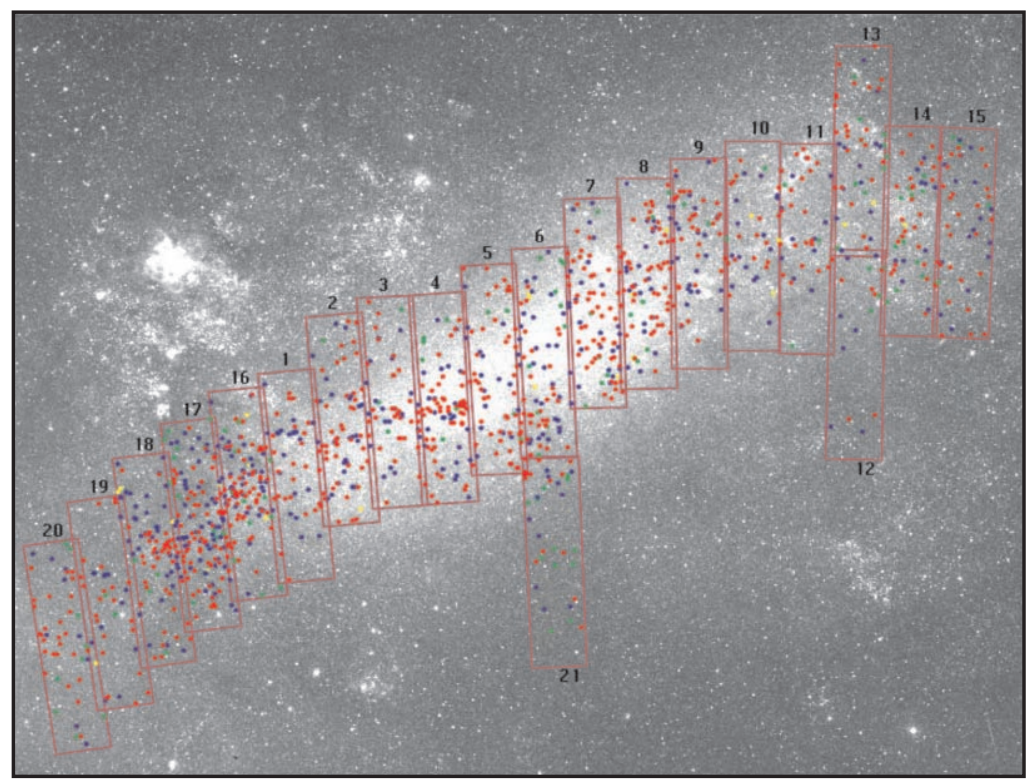

El gráfico de las estrellas de la Nube Mayor de Magallanes fue elaborado con el Software origin 8.

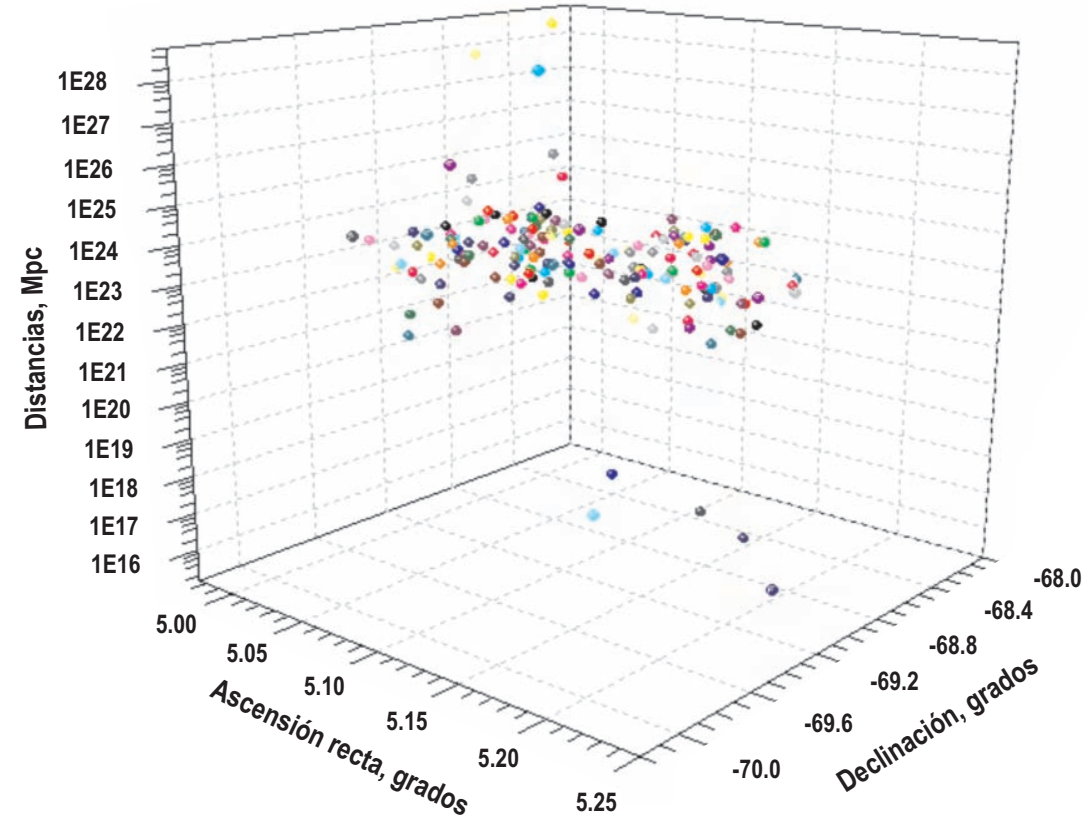

Fig.2: Gráfico tridimensional de la Nube Mayor de Magallanes 


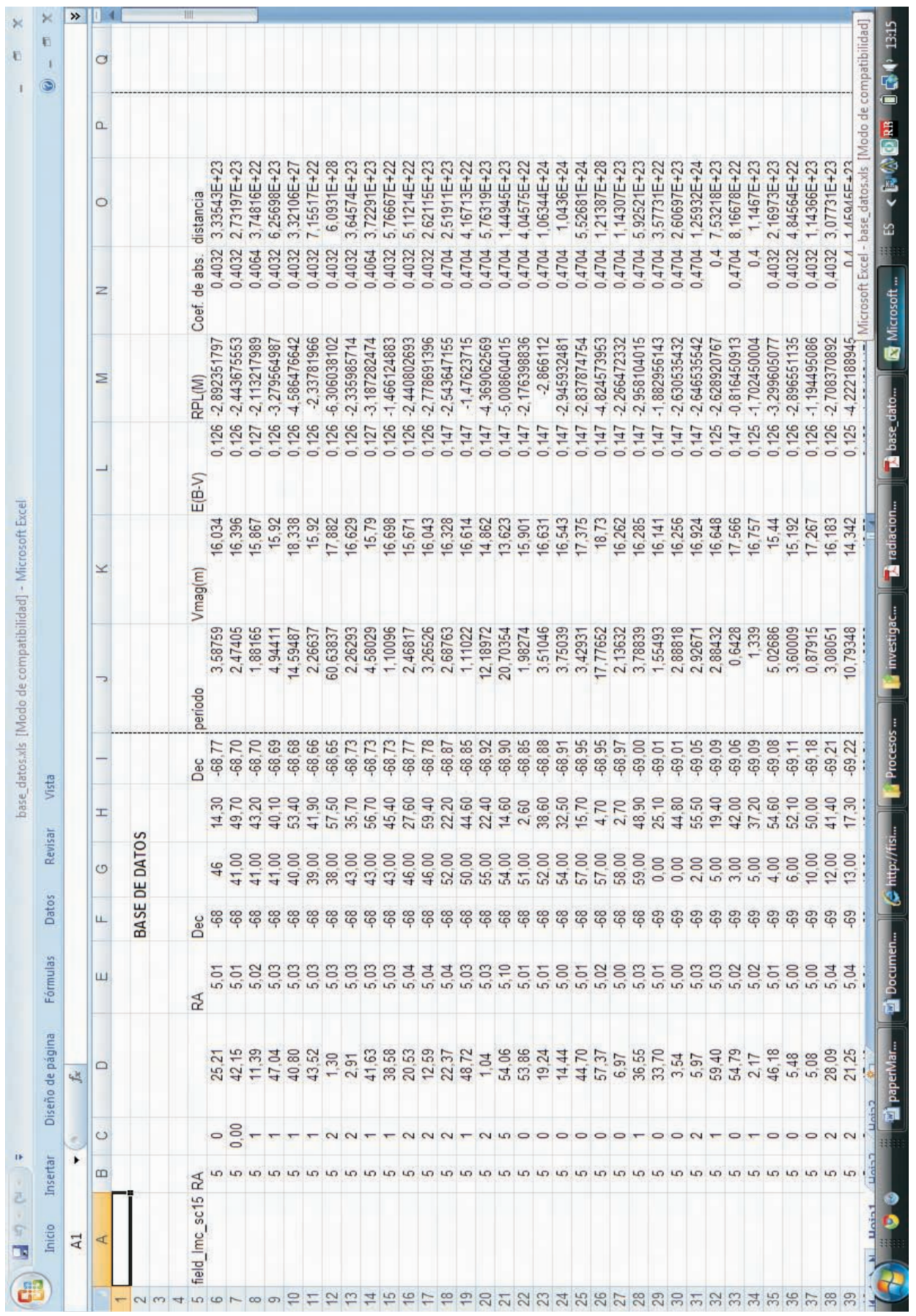

Fig.3 Base de datos de las estrellas seleccionadas 